\title{
Nucleic acid scavenging microfiber mesh inhibits trauma-induced inflammation and thrombosis
}

Jaewoo Lee $^{1}$, Jennifer G. Jackman ${ }^{2}$, Jean Kwun ${ }^{1,4}$, Miriam Manook ${ }^{1,4}$, Angelo Moreno ${ }^{3}$, Eric A. Elster ${ }^{5}$, Allan D. Kirk ${ }^{1,4}$, Kam W. Leong $^{2,6}$, Bruce A. Sullenger ${ }^{1,3} *$

1 Department of Surgery, Duke University, Durham, NC, 27710, USA

2 Department of Biomedical Engineering, Duke University, Durham, NC, 27710, USA

3 Department of Molecular Genetics and Microbiology, Duke University, Durham, NC 27710, USA

4 Duke Transplant Center, Department of Surgery, Duke University, Durham, NC, 27710, USA

5 Department of Surgery, Uniformed Services University of Health Sciences and Walter Reed National Military Medical Center, Bethesda, MD 20814, USA

6 Department of Biomedical Engineering, Columbia University, New York, NY, 10027, USA

* To whom correspondence should be addressed.

Tel: 011-1-919-684-6375; Fax:011-1-919-684-6492; Email: bruce.sullenegr@ duke.edu Tel: 011-1-919-613-5045; Fax:011-1-919-684-6492; Email: jaewoo.lee@duke.edu 


\begin{abstract}
Trauma patients produce a host of danger signals and high levels of damage-associated molecular patterns (DAMPs) after cellular injury and tissue damage. These DAMPs are directly and indirectly involved in the pathogenesis of various inflammatory and thrombotic complications in patients with severe injuries. No effective therapeutic agents for the removal of DAMPs from blood or tissue fluid have been developed. Herein, we demonstrated that nucleic acid binding polymers, e.g., polyethylenimine (PEI) and polyamidoamine dendrimers, immobilized onto electrospun microfiber mesh can effectively capture various DAMPs, such as extracellular DNAs and high mobility group box 1 (HMGB1). Furthermore, treatment with PEIimmobilized microfiber mesh abrogated the ability of DAMPs, released from dead and dying cells in culture or found in patients following traumatic injury, to activate innate immune responses and coagulation in vitro and in vivo. Nucleic acid scavenging microfiber meshes represent an effective strategy to combat inflammation and thrombosis in trauma.
\end{abstract}

\title{
Keywords
}

Nucleic acid scavenger, Toll like receptor, Inflammation, Thrombosis, Microfiber mesh 


\section{Introduction}

Damage associated molecular patterns (DAMPs) are molecules released from damaged cells or extracellular matrix. Upon injury of tissues, DAMPs are released into the blood and tissue fluid. These DAMPs play a key role in healing damaged tissues and protecting against infection [1]. They are recognized by pattern recognition receptors (PRRs) expressed in mammalian cells. Upon binding to DAMPs, PRRs trigger intracellular signal cascades and lead to activation of transcription factors, including nuclear factor- $\kappa \mathrm{B}(\mathrm{NF}-\kappa \mathrm{B})$, activator protein 1 (AP1) and interferon regulatory factor (IRF), which elicit expression of inflammatory cytokines, type I interferons (IFN) and IFN-inducible genes [2]. Elevated levels of plasma DAMPs have been observed in patients with traumatic injuries and were strongly correlated with the development of severe posttraumatic complications, e.g., systemic inflammatory response syndrome (SIRS), multiple organ dysfunction and thrombosis [3, 4].

Unlike other PRRs, toll-like receptors (TLRs) are membrane-associated proteins and predominantly expressed in immune and inflammatory cells [5]. A particular TLR is able to recognize a specific molecular pattern associated with a virus, bacteria and/or damaged tissues. For instance, TLRs 2, 4, 5, 6 and 11 bind to bacterial membrane-associated molecules (e.g., lipoprotein and lipopolysaccharide (LPS)), cellular membrane-associated molecules (e.g., heparan sulfate) and nuclear proteins (e.g., high mobility group box 1 protein (HMGB1) and histones), whereas TLRs 3, 7, 8 and 9 recognize bacterial, viral and cellular DNAs and RNAs [6].

TLRs act as the sentinel of the innate immune system that protects against pathogens. Inappropriate activation of TLRs, on the other hand, contributes to the development of a variety of diseases, including autoimmune and inflammatory diseases [7, 8], cardiovascular diseases [9], 
sepsis [10], neuronal diseases [11] and cancer [12]. Thus, TLRs are attractive therapeutic targets. Inhibition of single or dual TLRs using receptor antagonists has been demonstrated to attenuate disease progression in some pre-clinical studies [13-15]. However, the interconnectedness and redundancy of TLR signaling is likely to limit the therapeutic efficacy of single or dual TLR inhibitors.

In previous studies, we and others demonstrated that certain types of nucleic acid-binding polymers (NABPs) such as third-generation polyamidoamine dendrimer (PAMAM-G3) are able to neutralize the ability of cell-free DNAs and RNAs to activate nucleic acid-sensing TLRs (e.g., TLR3, TLR7, TLR8 and TLR9) [16] and blood coagulation [17, 18]. One potential concern with soluble NABP therapy is that NABPs show dose-dependent toxicity in vivo [19]. Since NABPs are originally designed to deliver genes and siRNAs into cells, they are readily uptaken by cells, damage intracellular organelles, and can induce cell death [20-22]. Moreover, the NABPs cannot neutralize the ability of non-nucleic acid DAMPs to induce immune stimulation [16]. Therefore, we sought to immobilize NABPs on microfiber meshes to limit systemic exposure and improve the ability of NABPs to remove additional DAMPs. We observed that NABPs immobilized onto electrospun microfiber mesh removed extracellular DNAs as well as non-nucleic acid DAMP HMGB1 and neutralized the ability of DAMPs generated by ex vivo cell culture or DAMPs circulating in the blood of trauma patients to stimulate multiple TLRs, e.g., TLRs 2, 3, 4 and 9, and coagulation in vitro and in vivo.

\section{Materials and methods}

\subsection{Generation of NABP-immobilized PSMA/polystyrene microfiber meshes}


Electrospinning is broadly used to form continuous fibers using a polymer solution with an applied voltage $[23,24]$. It has been demonstrated that poly(styrene-alt-maleic anhydride) (PSMA) polymers formed microfibrous meshes by electrospinning [25], and these meshes have been demonstrated to functionally immobilize large molecules, e.g., enzymes, by covalent conjugation [26]. NABPs were immobilized onto the electrospun PSMA microfiber meshes. To increase the durability of microfiber meshes, a mixture of PSMA and polystyrene copolymers was used to generate microfibers. PSMA (0.3 g) (Sigma, Saint Louis, MO) and polystyrene (0.4 g) (Sigma) were dissolved by constant stirring for $24 \mathrm{~h}$ at room temperature in a 1:1:1 (v:v:v) mixture of tetrahydrofuran:acetone:dimethylformamide (3 mL) (Sigma). Microfibers were generated by electrospinning $2 \mathrm{~mL}$ of copolymer solution using a 2cc glass syringe (Cadence Science, Staunton, VA) at a dispensing rate of $2 \mathrm{ml} / \mathrm{h}$ with an applied voltage of $\sim 17.3 \mathrm{kV}$, as described previously $[25,27]$. To generate microfiber meshes, the microfibers were collected on a grounded cylindrical mandrel ( $\sim 6.4 \mathrm{~cm}$ wide with a $\sim 21.6 \mathrm{~cm}$ circumstance) spinning at $\sim 130$ RPM at a distance of $17.3 \mathrm{~cm}$ away from the tip of the syringe needle. To generate NABPimmobilized meshes, the PSMA/polystyrene microfiber meshes were incubated with a solution of various NABPs in $\mathrm{dH}_{2} \mathrm{O}$, including $1.8 \mathrm{kDa}$ branched polyethylenimine (PEI) (Polysciences, Warrington, PA) $(0.005 \mathrm{M})$ and PAMAM-G3 $(0.004 \mathrm{M})$ (Sigma) for $72 \mathrm{~h}$ either at room temperature (PEI) or $4^{\circ} \mathrm{C}$ (PAMAM-G3) with constant shaking, washed 6 times for 10 min with $\mathrm{dH}_{2} \mathrm{O}$, and sterilized in $70 \%(\mathrm{v} / \mathrm{v})$ ethanol for $30 \mathrm{~min}$, followed by air drying. The levels of NABPs immobilized onto the PSAM/polystyrene meshes were determined by ninhydrin assay.

\subsection{Electron microscopy}

Dry PSMA/polystyrene meshes were placed on aluminum foil and mounted on a scanning electron microscope (SEM) stub. The filters were gold sputter-coated for $250 \mathrm{sec}$ using the 
Denton Vacuum Desk IV sputter unit (Denton Vacuum, Moorestown, NJ) and imaged using a FEI XL30 SEM-FEG (FEI, Hillsboro, OR). Images were analyzed using Scandium (ResAlta Research Technologies, Golden, CO).

\subsection{Cell culture}

Human normal lung fibroblast (ATCC, Manassas, VA) and human melanoma cell line WM266-4 (ATCC) was maintained in Eagle's Minimum Essential Medium supplemented with 10\% FBS, 1X non-essential Amino Acid and 1mM sodium pyruvate (all from Invitrogen, Carlsbad, CA). Human pancreatic cancer cell line PANC-1 (kindly provided by Dr. Rebekah White, Duke University, Durham, NC), mouse melanoma cell line B16-F10 (ATCC), mouse macrophage cell line RAW264.7 and mouse embryonic fibroblast (ATCC) were maintained in DMEM supplemented with 10\% FBS. TLR reporter cell lines, including HEK-hTLR3, HEKhTLR4 and HEK-hTLR9 cells (InvivoGen, San Diego, CA), stably express an NF-kB/AP-1inducible secreted embryonic alkaline phosphatase (SEAP) and corresponding TLR, and these reporter cells were maintained by following the manufacturer's instructions. All cells were incubated at $37^{\circ} \mathrm{C}$ in a humidified atmosphere with $5 \% \mathrm{CO}_{2}$.

\subsection{Quantification of cell growth}

Cell growth was quantified at $72 \mathrm{~h}$ after treatments using an Celltiter 96® MTS Cell Proliferation Assay Kit (Promega, Madison, WI), according to the manufacturer's instructions.

\subsection{Preparation of DAMPs}

DAMPs were isolated from ex vivo killed cells. To generate doxorubicin-induced cell death, $1 \times 10^{6}$ cells were incubated for $4 \mathrm{~h}$ with doxorubicin $(10 \mu \mathrm{M})$ (Sigma) followed by washing 5 times with fresh culture media. Cells were incubated for 2 days in $1 \mathrm{ml}$ of culture media. Culture 
supernatants were collected, centrifuged for $5 \mathrm{~min}$ at $1200 \mathrm{RPM}$ and stored at $-80^{\circ} \mathrm{C}$ until use. To generate sonication-induced cell death, $1 \times 10^{6}$ cells in $1 \mathrm{ml}$ of Dulbecco's phosphate-buffered saline (DPBS) (Sigma) were sonicated for 1.5 min with Branson Sonifier 250 (Branson Ultrasonics, Danbury, CT). The levels of extracellular DNAs (exDNAs), HMGB1, adenosine triphosphate (ATP) and uric acid in the DAMPs were determined using Quant-iT PicoGreen DNA assay kit (ThermoFisher, Waltham, MA), HMGB1 ELISA kit (Tecan, Morrisville, NC), ATP determination kit (ThermoFisher) and Uric Acid Assay kit (Sigma), respectively, by following the manufacturer's instructions. For circulating DAMPs in human blood, sera from citrated blood samples were collected from 3 patients with polytrauma and 3 normal healthy volunteers. The use of human blood samples was approved by the Institutional Review Board of Duke University Medical Center.

\subsection{Inhibition of DAMPs using NABP-immobilized meshes}

A NABP-immobilized mesh $\left(1.7 \times 1.7 \mathrm{~cm}\right.$; surface area of approximately $\left.2.9 \mathrm{~cm}^{2}\right)$ was inserted into each well of 12-well plate. $1 \mathrm{ml}$ of DAMPs were added into each well containing the NABP-immobilized mesh and incubated for $1 \mathrm{~min}$ at room temperature. Unbound DAMPs were harvested and added into a well containing a fresh NABP-immobilized mesh. To increase the dose of NABPs, the surface area of NABP-immobilized mesh was increased by repeated exposure of NABP-immobilized mesh to the DAMPs.

\subsection{Innate immune stimulation by DAMPs}

DAMPs with or without pre-treatment with NABP-immobilized meshes were diluted to $25 \%$ (v/v) with fresh complete media. TLR reporter cells were incubated with these DAMPs in a 96well plate. Upon binding to their cognate ligands, TLR signaling activates NF- $\mathrm{KB}$ which leads to expression and release SEAP from TLR reporter cells. The level of SEAP release was 
determined using a colorimetric assay. Briefly, after overnight incubation, $40 \mu \mathrm{l}$ culture supernatants were harvested and incubated for 3 to $5 \mathrm{~h}$ with $180 \mu \mathrm{l}$ QUANTI-Blue ${ }^{\mathrm{TM}}$ (InvivoGen) in a flat-bottom 96-well plate. SEAP activity was accessed by reading the optical density (OD) at $650 \mathrm{~nm}$ with BioTek Power Wave XS2 ELISA plate reader (BioTek, Winooski, VT). To stimulate mouse macrophage cells, RAW264.7 cells were incubated overnight with the DAMPs. Tumor necrosis factor (TNF)- $\alpha$ and interferon (IFN)- $\beta$ production by RAW264.7 cells was determined by enzyme-linked immunosorbent assay (ELISA) using BD OptEIA ${ }^{\mathrm{TM}}$ TNF- $\alpha$ ELISA sets (BD Biosciences, Franklin Lakes, NJ) and IFN- $\beta$ ELISA kit (PBL Biomedical Laboratories, Piscataway, NJ), respectively, by following the manufacturer's instructions. Unmethylated cytosine-phosphodiester-guanine oligodeoxynucleotide (CpG ODN) (5 $\mu \mathrm{M}$; TLR9 agonist) (InvivoGen), polyI:C (5 $\mu \mathrm{g} / \mathrm{ml}$; TLR3 agonist) (InvivoGen), Pam3CSK4 (100 ng/ml; TLR2 agonist) (InvivoGen), LPS (20 ng/ml; TLR4 agonist) (Sigma) and bovine kidney heparan sulfate (50 $\mu \mathrm{g} / \mathrm{ml}$; TLR4 agonist) (Sigma) were used as TLR stimulator controls. Untreated culture media and DPBS were used as negative controls.

\subsection{Plasma coagulation assay}

Human and mouse plasma coagulation assays were performed by described previously with minor modifications [17]. $5 \mu$ DAMPs with or without pre-treatment with NABP-immobilized meshes were added to $50 \mu \mathrm{l}$ normal pooled human plasma in sodium citrate (George King BioMedical Inc., Overland Park, KS) or normal pooled mouse plasma in sodium citrate (C57BL/6) (Biochemed services, Winchester, VA). The reaction was incubated for 3 min at $37^{\circ} \mathrm{C}$, followed by the addition of $50 \mu \mathrm{l} \mathrm{CaCl} 2(25 \mathrm{mM})$. Clotting times were recorded using STart ${ }^{\circledR}$ Hemostasis Analyzer (Diagnostica Stago, Parsippany, NJ). 


\subsection{Thromboelastography assay}

To test the efficiency of whole blood coagulation, Thromboelastography (TEG) assay was performed as previously described [17], using citrated whole mouse blood. Untreated mouse DAMPs or PEI mesh-treated mouse DAMPs were added to the citrated mouse blood, together with $\mathrm{CaCl}_{2}$. Time to clot formation ( $\mathrm{R}$ time, min) was recorded using TEG 500 (Haemoscope Corporation, Niles, IL). Fresh culture media and DPBS were used as negative controls.

\subsection{Heterotopic heart transplantation}

CBA (H-2k) mice (Jackson Laboratory, Bar Harbor, ME) were housed in a specific pathogen-free barrier facility and used at 6-12 weeks of age. Heterotopic heart transplantation was performed as previously described [28]. Briefly, the CBA recipient mouse was anesthetized with isoflurane. A segment of descending aorta and vena cava below the renal vessels was dissected. The heart was immediately removed from the CBA donor, flushed with $5 \mathrm{ml}$ of Eurocollins preservation solution and placed in chilled Eurocollins solution on ice. Before transplantation, $2 \mathrm{ml}$ of Eurocollins mixed with either mouse DAMPs or PEI mesh-treated mouse DAMPs was applied to the explanted donor heart via donor aorta and pulmonary artery. The CBA donor heart was then placed in the abdominal cavity of the recipient, and the donor aorta and pulmonary artery were anastomosed in an end-to-side manner to the recipient abdominal aorta and vena cava using 10-0 nylon suture. Acute thrombosis in the transplanted heart was monitored and recorded. The animals were sacrificed at $30 \mathrm{~min}$ after unclamping for histological analysis. The explanted grafts were sagittally bisected and fixed in $10 \%$ formalin. Paraffin embedded tissue was cut into $5 \mu \mathrm{m}$ sections and stained with trichrome-based staining (Carstairs) to highlight platelet, fibrin and erythrocytes. All experimental procedures involving the use of 
mice were performed in accordance with the guidelines and in compliance with the Animal Care and Use Committee of Duke University.

\subsection{Statistical analysis}

The paired two-tailed Student's t test was applied for determination of statistical significance. A probability of less than $0.05(\mathrm{P}<0.05)$ was used for statistical significance.

\section{Results}

3.1. NABP immobilized on a PSMA/polystyrene microfiber mesh inhibits nucleic acid and nonnucleic acid TLR ligands without adverse cytotoxic effects in cell culture

Non-woven electrospun PSMA/polystyrene microfibers were generated with an average diameter of $2.51 \pm 0.06 \mu \mathrm{m}$ (Fig. 1A). PEI or PAMAM-G3 serving as NABP was conjugated onto a 6.6x6.6 cm PSMA/polystyrene microfiber mesh. The potential of this NABP-immobilized PSMA/polystyrene microfiber meshes to inhibit the ability of nucleic acids to stimulate nucleic acid-recognizing TLRs, e.g., TLR3 and TLR9 was compared with unmodified PSMA/polystyrene microfiber mesh of free NABP in scavenging nucleic acid TLR agonists, such as polyI:C and CpG ODN. Consistent with our previous study [16], free PAMAM-G3 but not free PEI inhibited both polyI:C and CpG ODN to stimulate TLR3 and TLR9, respectively, but they could not inhibit non-nucleic acid TLR agonists, Pam3CSK4, LPS and heparan sulfate (Fig. 1B). PEI- and PAMAM-G3-immobilized meshes also inhibited TLR activation by CpG ODN and polyI:C. Unlike free PAMAM-G3, PEI- and PAMAM-G3-immobilized microfiber meshes inhibit heparan sulfate-mediated activation of TLRs but did not inhibit LPS- and Pam3CSK4-mediaetd activation of TLRs (Fig. 1B). Furthermore, as expected free PEI and free

PAMAM-G3 induced cytotoxicity of human fibroblasts in a dose-dependent manner (Fig. 1C 
and D). However, treatment with PEI- or PAMAM-G3-immobilized PSMA/polystyrene microfiber meshes did not engender significant cytotoxicity to various human and mouse cell lines and primary cells (Fig. 1E, F and Supplementary Fig. 1). These data demonstrate that NABPs immobilized on an electrospun PSMA/polystyrene microfiber mesh is able to inhibit both nucleic acid and certain non-nucleic acid TLR ligands without inducing significant cytotoxicity.

\subsection{NABP-immobilized microfiber mesh inhibits DAMP-and PAMP-mediated stimulation of} TLRS

To determine whether NABP-immobilized PSMA/polystyrene microfiber meshes are able to neutralize the ability of DAMPs to stimulate TLRs, we isolated DAMPs released from dead and dying human and mouse cells. The cells were killed by either sonication- or doxorubicin-induced cell death. DAMPs from either source activated both TLR4 and TLR9. The DAMPs treated with the PEI-immobilized microfiber meshes lost their ability to stimulate the TLR reporter cells in a surface size-dependent manner (Fig. 2A, B and C). Treatments with free PAMAM-G3 significantly, but weakly, inhibited the stimulation of TLR9 reporter cells but did not inhibit the stimulation of TLR4 reporter cells. Moreover, the free PAMAM-G3 was much less potent than the PEI-immobilized microfiber mesh. In addition, the PEI-immobilized microfiber meshes inhibited DAMP activation of mouse macrophages (Fig. 3D and E) and also significantly reduced the ability of bacterial PAMPs to stimulate multiple TLR reporter cells (Fig. 3F).

\subsection{NABP-immobilized microfiber mesh removes exDNA and HMGB1 from extracellular} compartments

Extracellular DNAs (exDNAs) and HMGB1 released from damaged cells are known as potent inflammatory and thrombotic mediators. The levels of exDNAs and HMGB1 are 
markedly elevated in the blood of patients with traumatic injuries compared with healthy controls [29, 30]. The exDNA and HMGB1 are endogenous ligands of TLR9 and TLR4, respectively, and they are associated with posttraumatic coagulation abnormalities, activation of complement and severe systemic inflammatory responses [31-33]. To determine whether NABPimmobilized PSMA/polystyrene microfiber mesh can capture and remove exDNA and HMGB1 from extracellular fluids, the levels of exDNA and HMGB1 released from ex vivo killed human cells were determined before and after treatment with various surface sizes of the PEIimmobilized PSMA/polystyrene microfiber mesh. Both exDNA and HMGB1 was significantly removed from the extracellular milieu by the PEI-immobilized PSMA/polystyrene mesh in a surface size-dependent manner (Fig. 3A and B). Interestingly, PEI-immobilized PSAM/polystyrene mesh did not remove ATP and uric acid, metabolic products released from dead and dying cells [34], from the extracellular fluids (Fig. 3C and D).

\subsection{NABP-immobilized microfiber mesh inhibits TLR stimulation by trauma patient DAMPs}

Next, we asked whether the NABP-immobilized meshes can counteract pro-inflammatory DAMPs in the blood of patients with traumatic injuries. DAMPs in sera isolated from the blood of trauma patients significantly increased stimulation of multiple TLR reporter cells, e.g., TLR2, TLR3, TLR4 and TLR9, compared with sera isolated from the blood of normal healthy volunteers (Fig. 4A). The TLR stimulatory activities of trauma patient DAMPs were significantly abrogated by treatment with PEI-immobilized meshes (Fig. 4B). Consistent with DAMPs generated by ex vivo cell death of human and mouse cells, treatment with PAMAM-G3, however, only marginally inhibited patient DAMP-stimulated TLR9 signaling, but did not inhibit patient DAMP-stimulated TLRs 2, 3 and 4 signaling. These data indicate that treatment with 
NABP-immobilized microfiber meshes can remove multiple immune stimulatory mediators from the blood of patients with traumatic injuries.

\subsection{PEI-immobilized microfiber mesh treatment prevents DAMP-induced thrombosis after heart} transplantation

Cell-free DNAs, RNAs and HMGB1 released from injured tissues are known as potent activators of coagulation and platelets [35-37]. Such activation has been associated with thrombotic complications and graft rejection following solid organ transplantation [38, 39]. Moreover, transplantations with lung from traumatic brain injury mice have been shown to develop HMGB1-mediated acute graft rejection [40]. To determine whether NABP-immobilized microfiber meshes could counteract DAMP-mediated coagulation and graft rejection, we performed in vitro coagulation and heart transplantation studies. DAMPs generated by sonication-induced human and mouse cells accelerated the clotting of human and mouse plateletdepleted plasmas (Fig. 5A and B). Treatments with PEI-immobilized microfiber meshes abrogated the ability of the DAMPs to activate plasma coagulation. Furthermore, the DAMPs dramatically accelerated the clotting of mouse whole blood (Fig. 5C). Consistent with plasma clotting analyses, the pre-treatment with PEI-immobilized microfiber meshes significantly reduced DAMP-induced whole blood coagulation. To access the pro-thrombotic effect of DAMPs in heart transplantation and anti-thrombotic effect of PEI-immobilized microfiber mesh in vivo, we treated donor hearts with DAMPs prior to transplantation. As shown in Figure 5D and F, donor hearts perfused with DAMPs rapidly developed occlusive thrombosis and stopped beating within 5 min after transplantation. In contrast, no acute thrombosis nor cessation of heart beating was observed in donor hearts from which DAMPs had been captured and removed for 10 minutes (Fig. 5E and F). 


\section{Discussion}

This study demonstrates that electrospun PSMA/polystyrene microfiber meshes functionalized with NABPs neutralized the ability of nucleic acid as well as non-nucleic acid DAMPs and PAMPs to stimulate TLRs and coagulation pathways. Since NABPs are immobilized on a solid surface, they do not circulate in the blood and enter cells. Therefore, the immobilized configuration of NABPs would be of particular interest to scavenge DAMPs and PAMPs in scenarios of tissue damage and inflammation. Nevertheless, this study also demonstrates that a NABP-immobilized microfiber mesh is able to remove DAMPs, such as exDNA and HMGB1, from the blood of trauma patients, thereby inhibiting DAMP-stimulated blood coagulation.

Because NABPs are highly positively charged, we initially speculated that all negatively charged DAMPs could be potential targets for the immobilized NABPs. NABPs immobilized on PSMA/polystyrene microfiber meshes inhibited negatively charged polymeric molecules such as DNA, RNA and heparan sulfate [41], but did not inhibit Pam3CSK4, a cationic amphiphilic lipopeptide, and anionic metabolic products (e.g., ATP and uric acid). Furthermore, the NABPimmobilized microfiber meshes captured and removed circulating HMGB1, a DNA-binding protein composed of two positively charged DNA-binding motifs and a C-terminal acidic tail [42]. This highly negatively charged C-terminal domain likely binds to NABPs. However, the NABP-immobilized microfiber meshes are unable to inhibit negatively charged LPS. Moreover, treatment with NABP-immobilized microfiber meshes did not change the growth supporting activity of serum-supplemented culture media. Serum contains a diverse spectrum of anionic proteins (e.g., serum albumin), anionic amino acids (e.g. aspartate and glutamate) and anionic nutrients that are required for survival and growth of cells. These data suggest that NABP- 
immobilized microfiber meshes may selectively bind to certain types of anionic DAMPs. Further studies are needed to elucidate the precise mechanisms by which NABP-immobilized microfiber meshes neutralize the ability of DAMPs to stimulate TLRs and induce inflammatory response and thrombosis.

We have previously demonstrated that a prototypic molecular scavenger PAMAM-G3 neutralized the ability of synthetic CpG DNA and polyI:C to stimulate TLRs and induce acute liver damage in mice [16]. However, pharmacokinetics and pharmacodynamics of PAMAM-G3 have not yet been determined. Moreover, many questions of mechanisms of action of PAMAMG3 remain to be answered, including whether the PAMAM-G3 inhibits DAMPs released from injured cells or tissues, how much negatively charged DAMPs are circulating in the blood of patients with injury, and how much DAMPs are needed to be inhibited by NABPs for the amelioration of inflammatory and thrombotic complications. Surprisingly, PAMAM-G3 marginally neutralized the ability of both DAMPs generated by death-induced cells and trauma patient sera to stimulate TLR9. The in vitro cell growth study suggested that the maximal tolerated dose of PAMAM-G3 was between 20 and $30 \mu \mathrm{g} / \mathrm{ml}$ (Fig. 1D). The levels of exDNAs released from death-induced cells were approximately $17 \mu \mathrm{g} / \mathrm{ml}$. The levels of exDNAs in the blood of trauma patients were varied between 1 and $16 \mu \mathrm{g} / \mathrm{ml}$ depending on disease severity [43]. Studies show that 200 to $600 \mathrm{ng} / \mathrm{ml}$ of HMGB1 is circulating in the blood of patients with traumatic injury [29]. Comparable amounts of exRNAs, other than anionic DAMPs and negatively charged membranous vesicles are simultaneously circulating in the blood of trauma patients $[44,45]$. Thus, the maximal tolerated doses of free PAMAM-G3 may be sub-optimal and lead to only marginal therapeutic effect. In contrast, either $288 \mu \mathrm{g} / \mathrm{cm}^{2}$ of PEI or $128 \mu \mathrm{g} / \mathrm{cm}^{2}$ of PAMAM-G3 can be immobilized onto the PSMA/polystyrene microfiber mesh and the dose 
of PEI and PAMAM-G3 immobilized onto the microfiber mesh are easily increased. Therefore, immobilized NABP is a more effective and safer therapeutic agent than free NABPs in this study.

DAMPs are potential biomarkers and attractive therapeutic targets for critically ill patients [46]. Cellular disruption by trauma releases DAMPs that are one of the key linkers between tissue damage, inflammation and systemic inflammatory response syndrome (SIRS) [32]. DAMPs influence not only disease progress in primary injured sites but also facilitate dysfunction of other organs and systemic complications [40]. Furthermore, negatively charged DAMPs, including hyaluronic acid, cell-free nucleic acids and heparan sulfate, released after allograft reperfusion induced inflammation and thrombosis, which have a negative impact on transplant outcomes [47] and facilitate pulmonary dysfunction and graft-versus-host disease after allogeneic transplantation [41]. Moreover, elevated circulating DAMPs were shown to correlate with the onset of septic shock and organ failure in patients with sepsis [48].

Extracorporeal membrane oxygenation (ECMO) and hemofilters are often used in critically ill patients who have cardiac and pulmonary dysfunctions and who have a high risk of acute lung and kidney injuries [49]. In a dog model, early treatments with continuous veno-venous hemofiltration $(\mathrm{CVVH})$ significantly decreased mortality and acute lung injury in animals with severe drowning accident [50]. The CVVH reduced the levels of circulating pro-inflammatory cytokines and oxidative stress in these animals. Moreover, blood purification by hemofilters is broadly used to remove circulating pathologic mediators from the patients with critical illness, such as severe sepsis and acute respiratory distress syndrome [51].

Depending on surface modifications, hemofilters can remove specific molecules from patient's blood. Cytokine-absorbing hemofilters decreased circulating pro-inflammatory 
cytokines, heart rate, blood lactate level, intra-abdominal pressure and mortality rate in patients with severe acute pancreatitis [52]. Fibers coated with endotoxin-binding polymyxin B [53, 54], opsonin [55, 56] or PEI [57] have been shown to remove endotoxin and pathogens from patient's blood and ameliorated sepsis and acute respiratory distress syndrome. However, no such hemofilters have been developed to remove DAMPs from the blood of patients with sterile inflammatory and thrombotic complications.

Certain types of DAMPs, including exDNAs and DNA-binding proteins (e.g., histone and HMGB1), are known to be potent pro-coagulants [58, 59]. Moreover, DAMP-stimulated TLRs on platelets and polymorphonuclear cells indirectly promote thrombosis [60,61]. Activation of TLR4 on platelets enhanced microvascular thrombosis in endotoxemia and trauma animal models [37, 62]. Patients with severe traumatic brain injury (TBI) have increased risk of disseminated intravascular coagulation $[63,64]$. Weber et al. demonstrated that TBI elevated the levels of circulating HMGB1 that caused systemic hypoxia, acute lung injury and pulmonary neutrophilia [40]. Lung transplantation from donor mouse with TBI induced acute pulmonary dysfunction, and treatments with HMGB1 neutralizing antibody ameliorated this pulmonary dysfunction [40]. Interestingly, in this study, a normal donor heart perfused with DAMPs developed acute thrombotic complications after transplantation, and treatment with NABPimmobilized microfiber meshes captured and removed DAMPs and prevented the development of thrombotic complications after transplantation (Fig. 5). Therefore, treatment with NABPimmobilized microfiber meshes can reduce systemic DAMP levels and ameliorate inflammatory and thrombotic complications in trauma and transplantation.

\section{Conclusions}


NABP-immobilized microfiber meshes have a potential use during ECMO, CVVH and continuous renal replacement therapy (CRRT) in intensive care units. Removing proinflammatory and pro-coagulative mediators from circulation is an unmet need in the treatment of critically ill patients. NABP-immobilized microfiber meshes can be developed as safe and effective anti-inflammatory and anti-thrombotic therapeutics for the treatment of patients with traumatic injuries and the enhancement of grafts of organs derived from patients with traumatic injuries.

\section{Acknowledgement}

This work was supported in part by Duke Department of Surgery (Clarence Gardner Award) (JL), Duke University Shared Materials Instrumentation Facility, a member of the North Carolina Research Triangle Nanotechnology Network, which is supported by the National Science Foundation (ECCS-1542015) as part of the National Nanotechnology Coordinated Infrastructure (JL), Pilot grant from the Opportunity Funds Management Core of the Centers for Medical Countermeasures against Radiation, National Institute of Allergy and Infectious

Diseases (U19AI067773) (JL), National Institutes of Health (NIH) Grant (U54HL112307, U19AI067798, 5R01HL065222) (BAS), NIH grant (HL109442, AI096305, GM110494) (KWL), Guangdong Innovative and Entrepreneurial Research Team Program (No.2013S086) (KWL) and the DoD/SC2i initiative: Department of Defense Health Program - Joint Program Committee 6 / Combat Casualty Care (USUHS HT9404-13-1-0032 and USUHS HU0001-15-2-0001) (ADK, EAE). We thank Dr. Dani Bolognesi for editing and critical comments on this manuscript.

\section{Disclosures}


We have no conflict of interest to declare.

\section{References}

[1] Venereau E, Ceriotti C, Bianchi ME. DAMPs from Cell Death to New Life. Frontiers in immunology. 2015;6:422.

[2] Kawai T, Akira S. TLR signaling. Seminars in immunology. 2007;19:24-32.

[3] Liaw PC, Ito T, Iba T, Thachil J, Zeerleder S. DAMP and DIC: The role of extracellular DNA and DNA-binding proteins in the pathogenesis of DIC. Blood reviews. 2015.

[4] Stoecklein VM, Osuka A, Lederer JA. Trauma equals danger--damage control by the immune system. J Leukoc Biol. 2012;92:539-51.

[5] Leulier F, Lemaitre B. Toll-like receptors--taking an evolutionary approach. Nat Rev Genet. 2008;9:165-78.

[6] Feldman N, Rotter-Maskowitz A, Okun E. DAMPs as mediators of sterile inflammation in aging-related pathologies. Ageing research reviews. 2015;24:29-39.

[7] Mills KH. TLR-dependent $\mathrm{T}$ cell activation in autoimmunity. Nat Rev Immunol. 2011;11:807-22.

[8] Suurmond J, Diamond B. Autoantibodies in systemic autoimmune diseases: specificity and pathogenicity. J Clin Invest. 2015;125:2194-202.

[9] Vallejo JG. Role of toll-like receptors in cardiovascular diseases. Clin Sci (Lond). 2011;121:1-10.

[10] Wiersinga WJ. Current insights in sepsis: from pathogenesis to new treatment targets. Curr Opin Crit Care. 2011;17:480-6. 
[11] Okun E, Griffioen KJ, Mattson MP. Toll-like receptor signaling in neural plasticity and disease. Trends Neurosci. 2011;34:269-81.

[12] Pradere JP, Dapito DH, Schwabe RF. The Yin and Yang of Toll-like receptors in cancer. Oncogene. 2014;33:3485-95.

[13] Barrat FJ, Meeker T, Chan JH, Guiducci C, Coffman RL. Treatment of lupus-prone mice with a dual inhibitor of TLR7 and TLR9 leads to reduction of autoantibody production and amelioration of disease symptoms. European journal of immunology. 2007;37:3582-6.

[14] Fenhammar J, Rundgren M, Hultenby K, Forestier J, Taavo M, Kenne E, et al. Renal effects of treatment with a TLR4 inhibitor in conscious septic sheep. Critical care. 2014;18:488.

[15] Takemura N, Kawasaki T, Kunisawa J, Sato S, Lamichhane A, Kobiyama K, et al. Blockade of TLR3 protects mice from lethal radiation-induced gastrointestinal syndrome. Nature communications. 2014;5:3492.

[16] Lee J, Sohn JW, Zhang Y, Leong KW, Pisetsky D, Sullenger BA. Nucleic acid-binding polymers as anti-inflammatory agents. Proceedings of the National Academy of Sciences of the United States of America. 2011;108:14055-60.

[17] Jain S, Pitoc GA, Holl EK, Zhang Y, Borst L, Leong KW, et al. Nucleic acid scavengers inhibit thrombosis without increasing bleeding. Proceedings of the National Academy of Sciences of the United States of America. 2012;109:12938-43.

[18] Smith SA, Choi SH, Collins JN, Travers RJ, Cooley BC, Morrissey JH. Inhibition of polyphosphate as a novel strategy for preventing thrombosis and inflammation. Blood. 2012;120:5103-10. 
[19] Labieniec-Watala M, Watala C. PAMAM dendrimers: destined for success or doomed to fail? Plain and modified PAMAM dendrimers in the context of biomedical applications. J Pharm Sci. 2015;104:2-14.

[20] Malik N, Wiwattanapatapee R, Klopsch R, Lorenz K, Frey H, Weener JW, et al. Dendrimers: relationship between structure and biocompatibility in vitro, and preliminary studies on the biodistribution of 125I-labelled polyamidoamine dendrimers in vivo. Journal of controlled release : official journal of the Controlled Release Society. 2000;65:133-48.

[21] Mukherjee SP, Lyng FM, Garcia A, Davoren M, Byrne HJ. Mechanistic studies of in vitro cytotoxicity of poly(amidoamine) dendrimers in mammalian cells. Toxicology and applied pharmacology. 2010;248:259-68.

[22] Mukherjee SP, Byrne HJ. Polyamidoamine dendrimer nanoparticle cytotoxicity, oxidative stress, caspase activation and inflammatory response: experimental observation and numerical simulation. Nanomedicine : nanotechnology, biology, and medicine. 2013;9:202-11.

[23] Dzenis Y. Material science. Spinning continuous fibers for nanotechnology. Science. 2004;304:1917-9.

[24] Bhardwaj N, Kundu SC. Electrospinning: a fascinating fiber fabrication technique. Biotechnology advances. 2010;28:325-47.

[25] Tang C, Ye, S., Liu, H. Electrospinning of poly(styrene-co-maleic anhydride) (SMA) and water-swelling behavior of crosslinked/hydrolyzed SMA hydrogel nanofibers. Polymer. 2007;48:4482-91.

[26] Stoilova O, Ignatova, M., Manolova, N., Godjevargova, T., Mita, D. G., Rashkov, I. Functionalized electrospun mats from styrene-maleic anhydride copolymers for immobilization of acetylcholinesterase. European Polymer Journal. 2010;46:1966-74. 
[27] Ignatova M, Stoilova O, Manolova N, Markova N, Rashkov I. Electrospun mats from styrene/maleic anhydride copolymers: modification with amines and assessment of antimicrobial activity. Macromolecular bioscience. 2010;10:944-54.

[28] Kwun J, Hu H, Schadde E, Roenneburg D, Sullivan KA, DeMartino J, et al. Altered distribution of $\mathrm{H} 60$ minor $\mathrm{H}$ antigen-specific CD8 T cells and attenuated chronic vasculopathy in minor histocompatibility antigen mismatched heart transplantation in Cxcr3-/- mouse recipients. J Immunol. 2007;179:8016-25.

[29] Peltz ED, Moore EE, Eckels PC, Damle SS, Tsuruta Y, Johnson JL, et al. HMGB1 is markedly elevated within 6 hours of mechanical trauma in humans. Shock. 2009;32:17-22.

[30] Simmons JD, Lee YL, Mulekar S, Kuck JL, Brevard SB, Gonzalez RP, et al. Elevated levels of plasma mitochondrial DNA DAMPs are linked to clinical outcome in severely injured human subjects. Ann Surg. 2013;258:591-6; discussion 6-8.

[31] Cohen MJ, Brohi K, Calfee CS, Rahn P, Chesebro BB, Christiaans SC, et al. Early release of high mobility group box nuclear protein 1 after severe trauma in humans: role of injury severity and tissue hypoperfusion. Crit Care. 2009;13:R174.

[32] Zhang Q, Raoof M, Chen Y, Sumi Y, Sursal T, Junger W, et al. Circulating mitochondrial DAMPs cause inflammatory responses to injury. Nature. 2010;464:104-7.

[33] Gando S, Otomo Y. Local hemostasis, immunothrombosis, and systemic disseminated intravascular coagulation in trauma and traumatic shock. Critical care. 2015;19:72.

[34] Garg AD, Martin S, Golab J, Agostinis P. Danger signalling during cancer cell death: origins, plasticity and regulation. Cell Death Differ. 2014;21:26-38. 
[35] Kannemeier C, Shibamiya A, Nakazawa F, Trusheim H, Ruppert C, Markart P, et al. Extracellular RNA constitutes a natural procoagulant cofactor in blood coagulation. Proceedings of the National Academy of Sciences of the United States of America. 2007;104:6388-93.

[36] Swystun LL, Mukherjee S, Liaw PC. Breast cancer chemotherapy induces the release of cell-free DNA, a novel procoagulant stimulus. Journal of thrombosis and haemostasis : JTH. 2011;9:2313-21.

[37] Vogel S, Bodenstein R, Chen Q, Feil S, Feil R, Rheinlaender J, et al. Platelet-derived HMGB1 is a critical mediator of thrombosis. J Clin Invest. 2015;125:4638-54.

[38] Slegtenhorst BR, Dor FJ, Rodriguez H, Voskuil FJ, Tullius SG. Ischemia/reperfusion Injury and its Consequences on Immunity and Inflammation. Curr Transplant Rep. 2014;1:147-54.

[39] Sayah DM, Mallavia B, Liu F, Ortiz-Munoz G, Caudrillier A, DerHovanessian A, et al. Neutrophil extracellular traps are pathogenic in primary graft dysfunction after lung transplantation. Am J Respir Crit Care Med. 2015;191:455-63.

[40] Weber DJ, Gracon AS, Ripsch MS, Fisher AJ, Cheon BM, Pandya PH, et al. The HMGB1RAGE axis mediates traumatic brain injury-induced pulmonary dysfunction in lung transplantation. Science translational medicine. 2014;6:252ra124.

[41] Brennan TV, Lin L, Huang X, Cardona DM, Li Z, Dredge K, et al. Heparan sulfate, an endogenous TLR4 agonist, promotes acute GVHD after allogeneic stem cell transplantation. Blood. 2012;120:2899-908.

[42] Belgrano FS, de Abreu da Silva IC, Bastos de Oliveira FM, Fantappie MR, Mohana-Borges R. Role of the acidic tail of high mobility group protein B1 (HMGB1) in protein stability and DNA bending. PLoS One. 2013;8:e79572. 
[43] Avriel A, Paryente Wiessman M, Almog Y, Perl Y, Novack V, Galante O, et al. Admission cell free DNA levels predict 28-day mortality in patients with severe sepsis in intensive care. PLoS One. 2014;9:e100514.

[44] Park MS, Owen BA, Ballinger BA, Sarr MG, Schiller HJ, Zietlow SP, et al. Quantification of hypercoagulable state after blunt trauma: microparticle and thrombin generation are increased relative to injury severity, while standard markers are not. Surgery. 2012;151:831-6.

[45] Fox A, Gal S, Fisher N, Smythe J, Wainscoat J, Tyler MP, et al. Quantification of circulating cell-free plasma DNA and endothelial gene RNA in patients with burns and relation to acute thermal injury. Burns. 2008;34:809-16.

[46] Timmermans K, Kox M, Scheffer GJ, Pickkers P. Danger in the Intensive Care Unit: Damps in Critically Ill Patients. Shock. 2016;45:108-16.

[47] Braza F, Brouard S, Chadban S, Goldstein DR. Role of TLRs and DAMPs in allograft inflammation and transplant outcomes. Nat Rev Nephrol. 2016;12:281-90.

[48] Timmermans K, Kox M, Scheffer GJ, Pickkers P. Plasma Nuclear and Mitochondrial Dna Levels, and Markers of Inflammation, Shock, and Organ Damage in Patients with Septic Shock. Shock. 2015.

[49] Chen H, Yu RG, Yin NN, Zhou JX. Combination of extracorporeal membrane oxygenation and continuous renal replacement therapy in critically ill patients: a systematic review. Critical care. 2014;18:675.

[50] Chen J, Chen G, Xiao D, Peng W, Yu G, Lin Y, et al. Continuous venovenous hemofiltration decreases mortality and ameliorates acute lung injury in canine model of severe salt water drowning. Scand J Trauma Resusc Emerg Med. 2016;24:40. 
[51] Hirasawa H. Indications for blood purification in critical care. Contrib Nephrol. 2010;166:21-30.

[52] Abe R, Oda S, Shinozaki K, Hirasawa H. Continuous hemodiafiltration using a polymethyl methacrylate membrane hemofilter for severe acute pancreatitis. Contrib Nephrol. 2010;166:5463.

[53] Ito M, Kase H, Shimoyama O, Takahashi T. Effects of polymyxin B-immobilized fiber using a rat cecal ligation and perforation model. ASAIO J. 2009;55:246-50.

[54] Binh NG, Manabe T, Co DX, Tuan ND, Thach PT, Kudo K. Polymyxin-B-immobilizedfiber column hemoperfusion with oseltamivir treatment for ARDS due to influenza H1N1/09. Respirol Case Rep. 2015;3:57-60.

[55] Kang JH, Super M, Yung CW, Cooper RM, Domansky K, Graveline AR, et al. An extracorporeal blood-cleansing device for sepsis therapy. Nature medicine. 2014;20:1211-6.

[56] Didar TF, Cartwright MJ, Rottman M, Graveline AR, Gamini N, Watters AL, et al. Improved treatment of systemic blood infections using antibiotics with extracorporeal opsonin hemoadsorption. Biomaterials. 2015;67:382-92.

[57] Shum HP, Chan KC, Kwan MC, Yan WW. Application of endotoxin and cytokine adsorption haemofilter in septic acute kidney injury due to Gram-negative bacterial infection. Hong Kong Med J. 2013;19:491-7.

[58] Gould TJ, Vu TT, Swystun LL, Dwivedi DJ, Mai SH, Weitz JI, et al. Neutrophil extracellular traps promote thrombin generation through platelet-dependent and plateletindependent mechanisms. Arterioscler Thromb Vasc Biol. 2014;34:1977-84.

[59] Liaw PC, Ito T, Iba T, Thachil J, Zeerleder S. DAMP and DIC: The role of extracellular DNA and DNA-binding proteins in the pathogenesis of DIC. Blood Rev. 2016;30:257-61. 
[60] Fuchs TA, Brill A, Duerschmied D, Schatzberg D, Monestier M, Myers DD, Jr., et al. Extracellular DNA traps promote thrombosis. Proc Natl Acad Sci U S A. 2010;107:15880-5.

[61] Maugeri N, Campana L, Gavina M, Covino C, De Metrio M, Panciroli C, et al. Activated platelets present high mobility group box 1 to neutrophils, inducing autophagy and promoting the extrusion of neutrophil extracellular traps. Journal of thrombosis and haemostasis : JTH. 2014;12:2074-88.

[62] Stark RJ, Aghakasiri N, Rumbaut RE. Platelet-derived Toll-like receptor 4 (Tlr-4) is sufficient to promote microvascular thrombosis in endotoxemia. PLoS One. 2012;7:e41254.

[63] Sun Y, Wang J, Wu X, Xi C, Gai Y, Liu H, et al. Validating the incidence of coagulopathy and disseminated intravascular coagulation in patients with traumatic brain injury--analysis of 242 cases. Br J Neurosurg. 2011;25:363-8.

[64] Gando S. Hemostasis and thrombosis in trauma patients. Semin Thromb Hemost. 2015;41:26-34. 


\section{Figure legends}

Fig. 1. NABP-immobilized PSMA/polystyrene microfiber mesh scavenges multiple TLR agonists without cytotoxicity. A. scanning electron microscope (SEM) image of surface of PSMA/polystyrene microfiber mesh. B. Complete culture media (1 ml) supplemented with TLR agonists Pam3CSK4, LPS, Heparan sulfate (HS), polyI:C or CpG ODN, were incubated for 1 min with or without either PEI- or PAMAM-G3-immobilized microfiber mesh $\left(2.9 \mathrm{~cm}^{2}\right)$ at room temperature. The treatment was repeated once. TLR reporter cells were incubated for 3 days in either untreated or treated complete culture media. For the treatment with free NABPs, TLR reporter cells in the complete culture media containing fetal bovine serum and TLR agonist were directly treated with either PEI $(20 \mu \mathrm{g} / \mathrm{ml})$ or PAMAM-G3 $(25 \mu \mathrm{g} / \mathrm{ml})$. The activation of NF-kB in TLR signaling pathway was determined by a colorimetric enzyme assay. C-F. Human primary fibroblasts were cultured in either complete culture media containing free (C) PEI or free (D) PAMAM-G3 at various concentrations or complete culture media pre-treated with (E) PEIimmobilized mesh or (F) PAMAM-G3-immobilized mesh at various surface areas $(0.288$ $\mathrm{mg} / \mathrm{cm}^{2}$ PEI on mesh; $0.128 \mathrm{mg} / \mathrm{cm}^{2}$ PAMAM-G3 on mesh). After 3 days incubation, cell proliferation was determined by a MTS (3-(4,5-dimethylthiazol-2-yl)-5-(3carboxymethoxyphenyl)-2-(4-sulfophenyl)-2H-tetrazolium) assay. Error bars are S.D.. * Significant different $(P<0.05)$, vs untreated group.

Fig. 2. Inhibition of DAMP and PAMP-mediated TLR activation by PEI-immobilized PSMA/polystyrene microfiber mesh. A, B. DAMPs were generated from human and mouse cells 
that were killed by either sonication or doxorubicin. (A) TLR4 and (B) TLR9 reporter cell lines were stimulated overnight with human DAMPs $(20 \% \mathrm{v} / \mathrm{v})$ with or without treatment with either PEI-immobilized mesh $\left(5.8 \mathrm{~cm}^{2}\right)$ or PAMAM-G3 $(25 \mu \mathrm{g} / \mathrm{ml})$. C. TLR9 reporter cells were stimulated with DAMPs pre-treated with PEI-immobilized mesh at various surface sizes. The activation of NF-kB in TLR signaling pathway was determined by a colorimetric enzyme assay. D, E. Mouse macrophage cell line was incubated overnight with mouse DAMPs pre-treated with PEI-immobilized DAMPs at various surface sizes. (D) TNF- $\alpha$ and (E) IFN- $\beta$ production by the cells were determined by ELISA. F. Bacterial PAMPs were pre-treated with or without PEIimmobilized mesh $\left(5.8 \mathrm{~cm}^{2}\right)$. TLR3, TLR4 and TLR9 reporter cell lines were stimulated overnight with the untreated or treated PAMPs $(5 \% \mathrm{v} / \mathrm{v})$. Error bars are S.D.. * Significant different $(P<0.05)$, between indicated groups or compared with untreated.

Fig. 3. PEI-immobilized PSAM/polystyrene microfiber mesh capture and remove exDNA and HMGB1. Human DAMPs generated by sonication-induced cell death were treated with PEIimmobilized PSAM/polystyrene meshes at various surface sizes. The levels of (A) exDNAs, (B) HMGB1, (C) ATP and (D) uric acid in the DAMPs were determined by PicoGreen assay, ELISA, Bioluminescence assay and Fluorometric assay, respectively. Error bars are S.D.. * Significant different $(P<0.05)$, vs Untreated. $* *$ Significant different $(P<0.01)$, vs Untreated.

Fig. 4. PEI-immobilized microfiber mesh inhibits pro-inflammatory DAMPs from trauma patients. A. TLR2, TLR3, TLR4 and TLR9 reporter cells were incubated overnight with sera 
$(20 \% \mathrm{v} / \mathrm{v})$ isolated from either trauma patients or normal healthy volunteers. B. Undiluted serum was treated with or without either PEI-immobilized microfiber mesh $\left(8.7 \mathrm{~cm}^{2}\right)$ or PAMAM-G3 (25 $\mu \mathrm{g} / \mathrm{ml})$. NF- $\mathrm{\kappa B}$ activation in the TLR reporter cell lines was determined by a colorimetric assay. Error bars are S.D.. $* P<0.05 .{ }^{*} P<0.01$.

Fig. 5. Inhibition of DAMP-induced clotting by PEI-immobilized PSAM/polystyrene microfiber mesh. Human and mouse sonication-induced DAMPs were incubated for $1 \mathrm{~min}$ with or without PEI-immobilized PSAM/polystyrene microfiber meshes $\left(2.9 \mathrm{~cm}^{2}\right)$. The treatment was repeated four times. A, B. Platelet-depleted plasma from human and mouse normal blood were incubated with the DAMPs $(10 \% \mathrm{v} / \mathrm{v})$ and the clotting time measured. C. Mouse whole blood was incubated with the DAMPs at various dilutions with PBS. The coagulation (R) time was detected by Thromboelastography (TEG). D, E. Donor hearts isolated from normal mice $(n=3)$ was perfused with the DAMP (2 ml), followed by heterotrophic heart transplantation. Heart beating and thrombosis of donor heart was monitored. The image was captured 30 min after unclamping. F. Representative sections from untreated, DAMP-treated, and PEI-immobilized mesh-filtered DAMP-treated allografts harvested $30 \mathrm{~min}$ after unclamping. All were stained with Carstairs (modified Masson's trichrome) to visualize platelets (purple), erythrocytes (clear yellow/orange), and fibrin (Bright red/orange) taken at x100 magnification. Data represent three independent experiments. Error bars are S.D.. NS: statistically non-significant. $* P<0.05 . * * P<0.01$. 
Figure 1
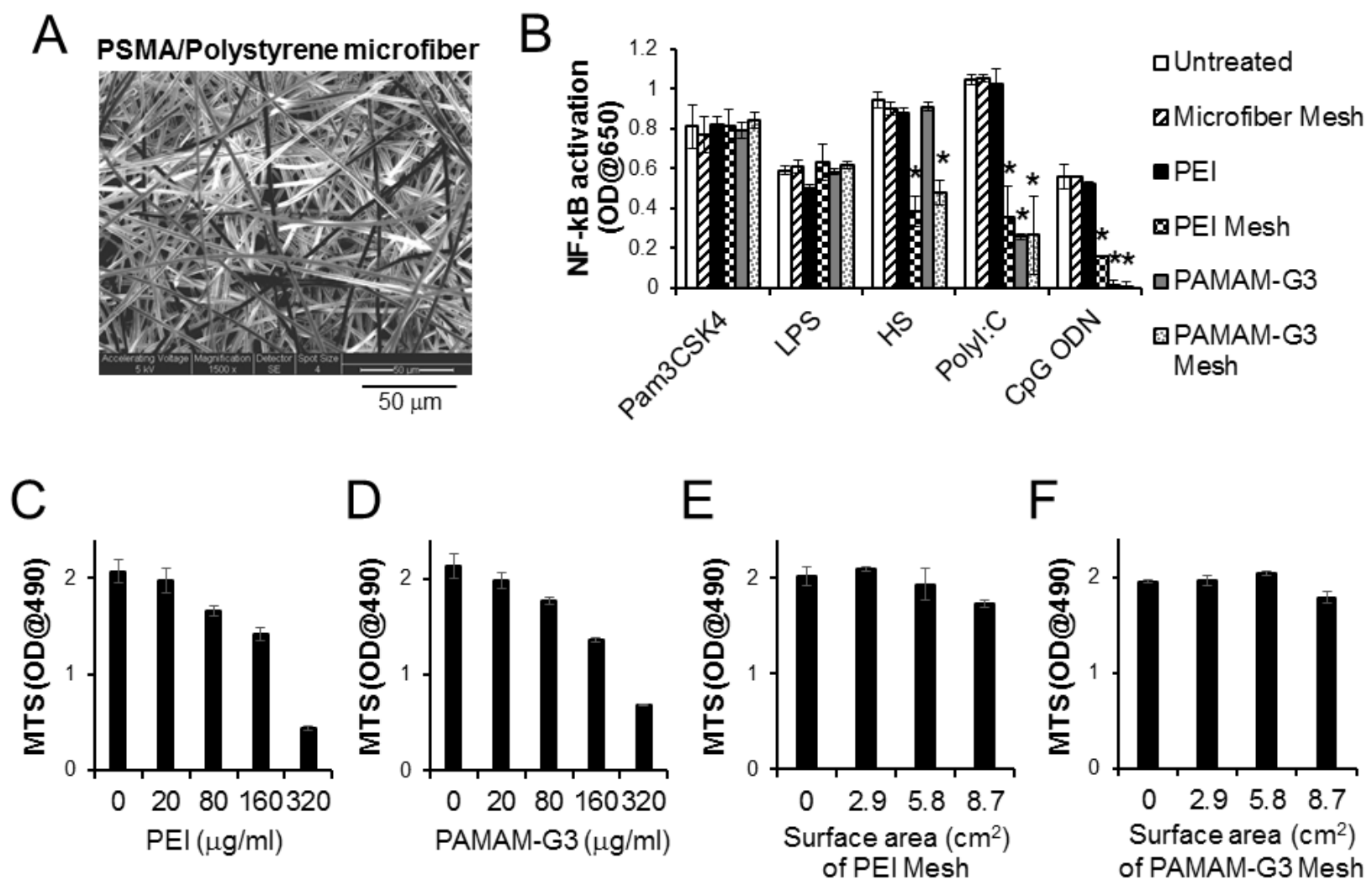
Figure 2

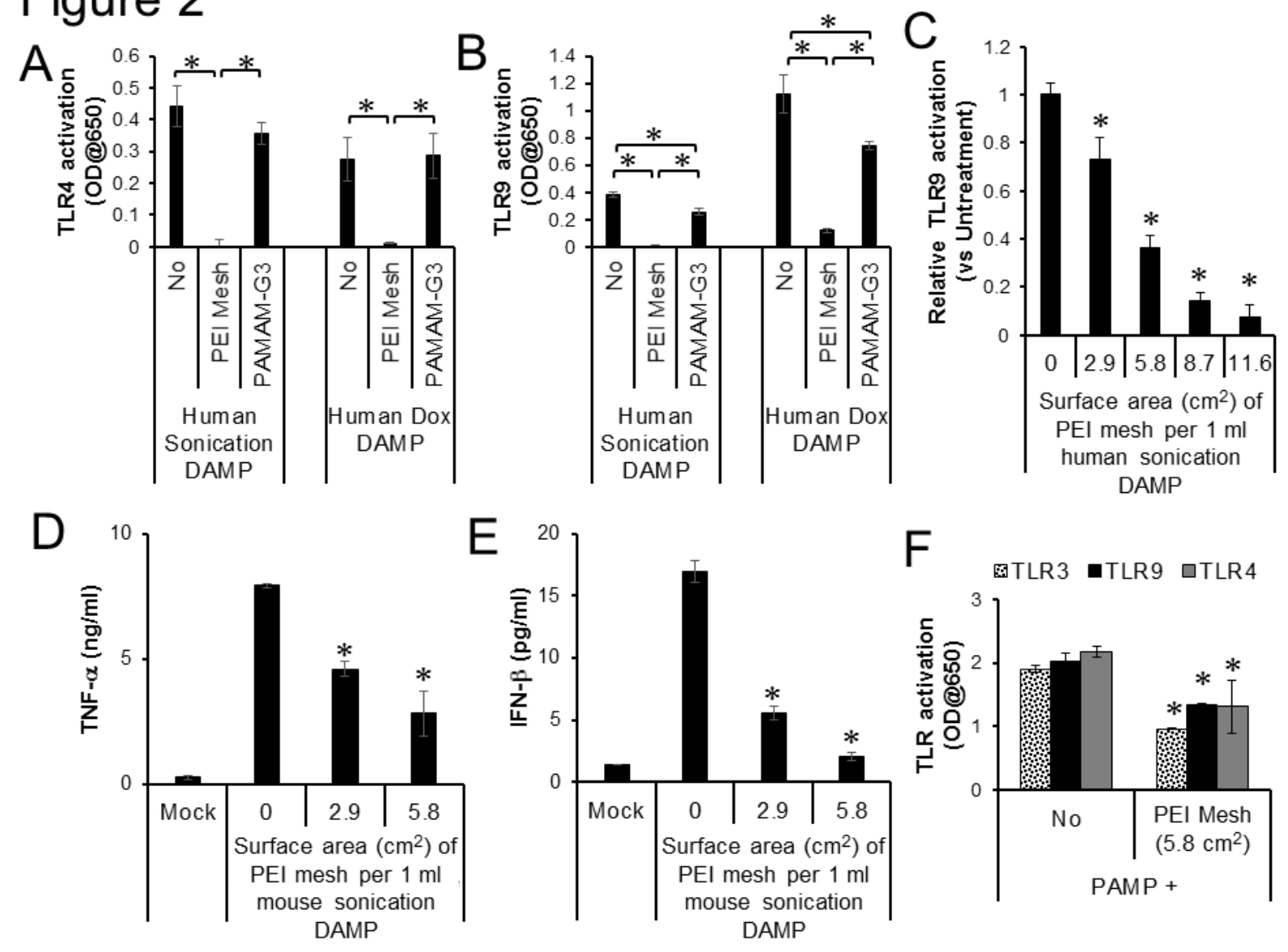


Figure 3
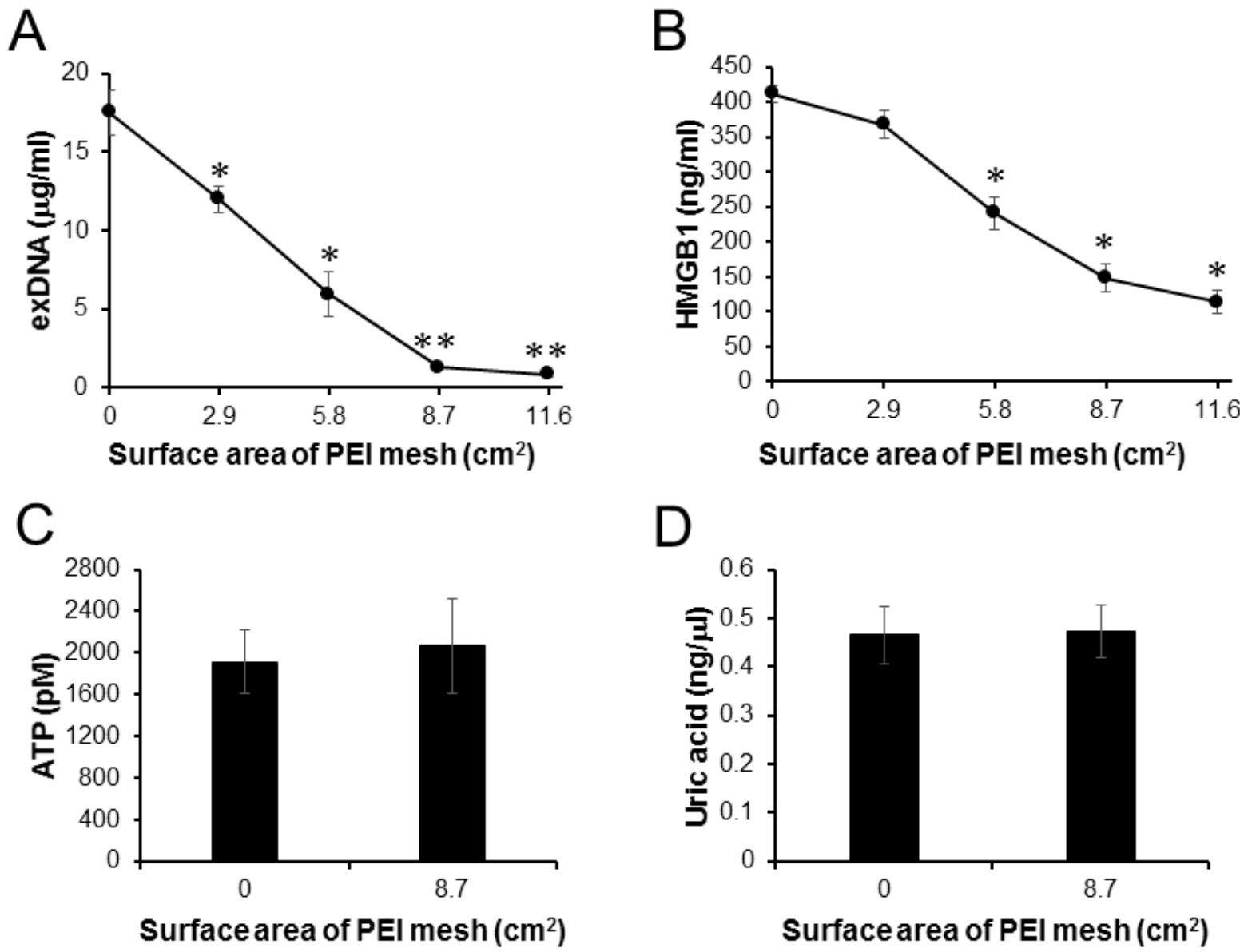

D

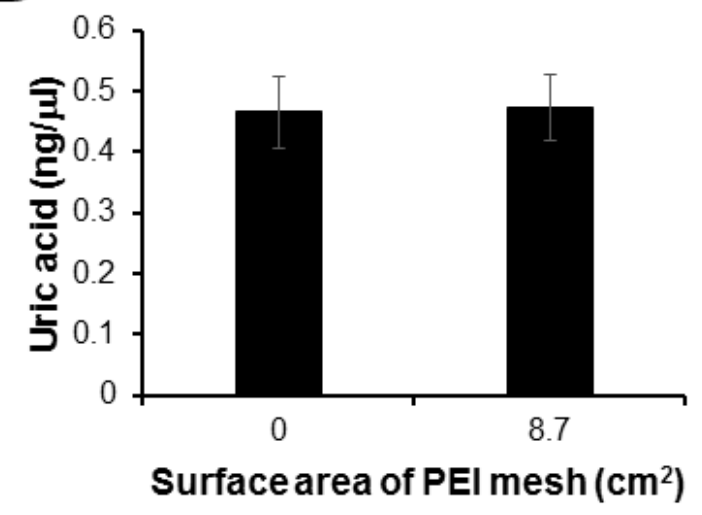


Figure 4

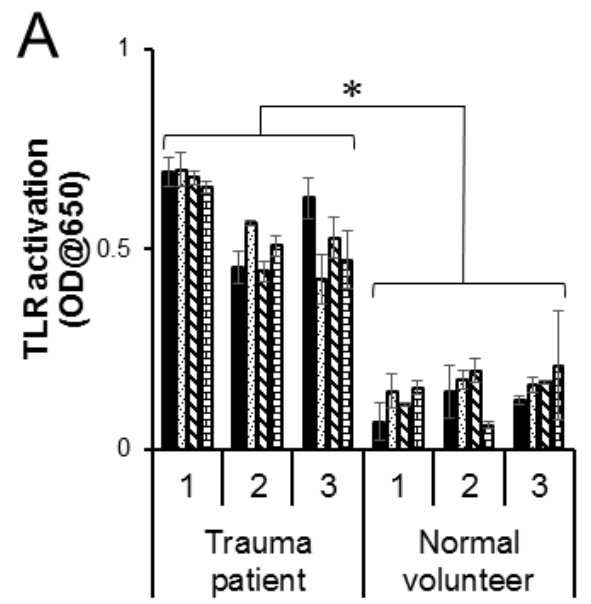

B

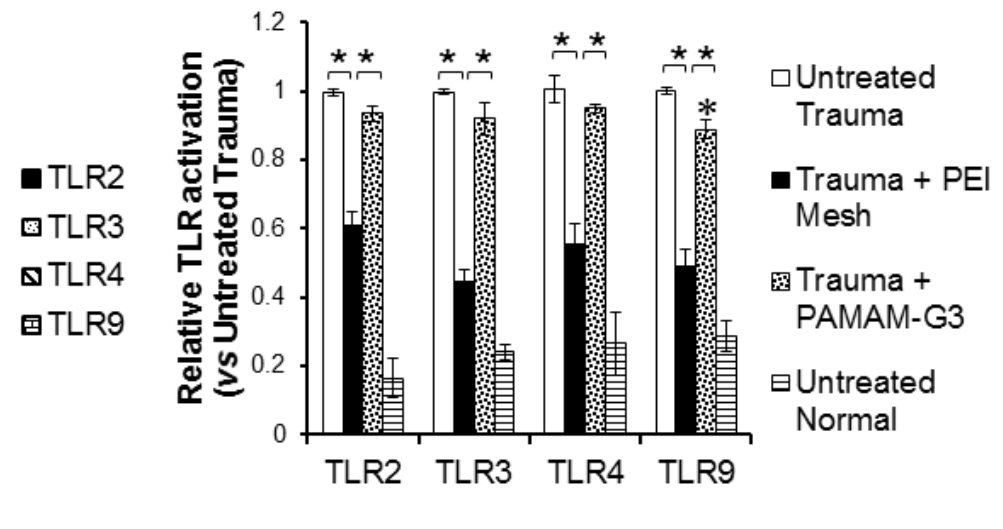


Figure 5
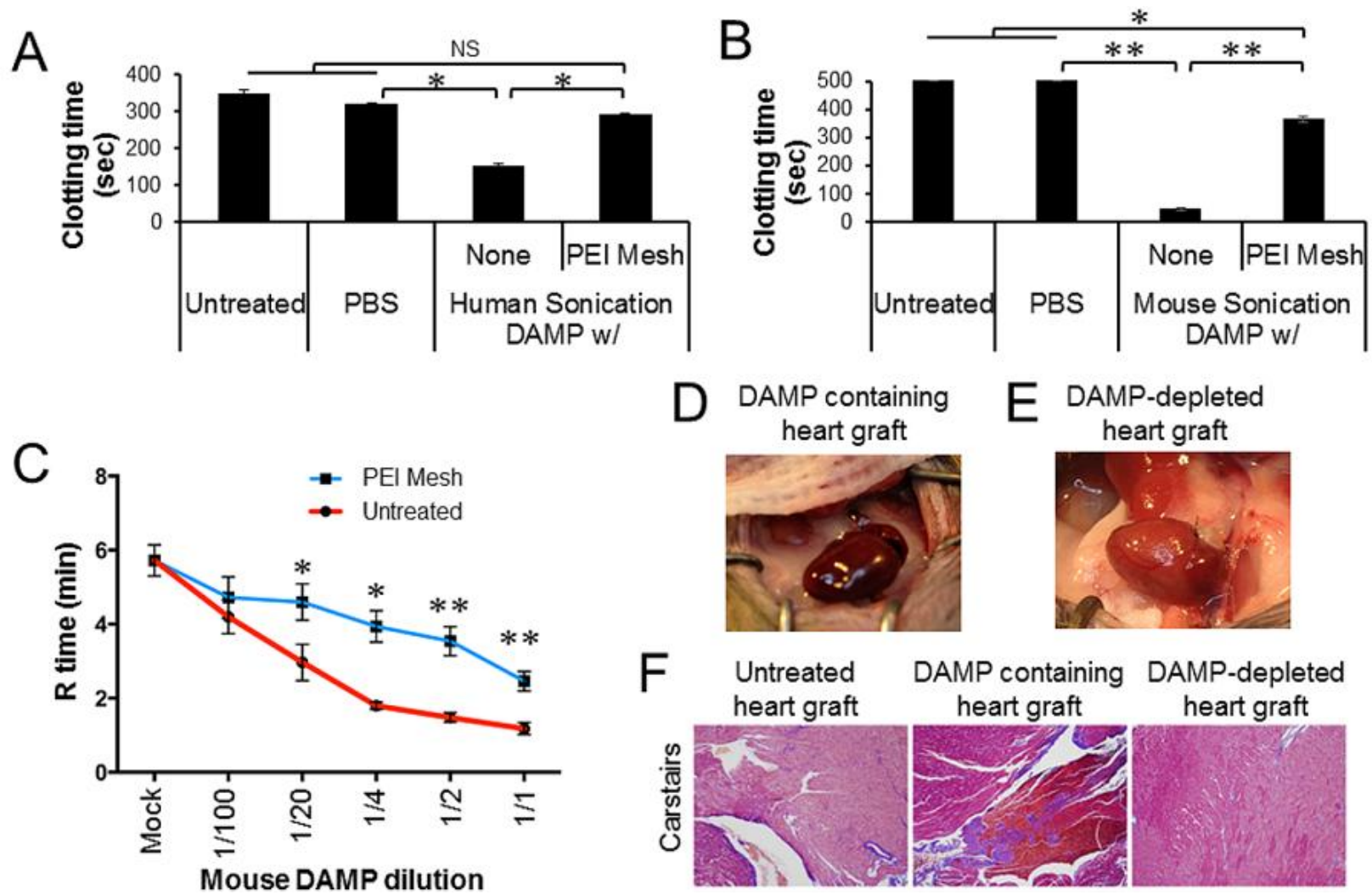